\title{
Linking HSCs to their youth
}

\section{Eric M. Pietras and Emmanuelle Passegué}

Nat. Cell Biol. 15, 885-887 (2013); published online 1 August 2013; corrected after print 2 August 2013

In the version of this News and Views originally published, there was an error in Fig. 1: the blue cell on the right should have been labelled 'Adult HSC', not 'Fetal HSC'. This has been corrected in the HTML and PDF versions. 